\title{
Assessment of repeatability and treatment response in early phase clinical trials using DCE-MRI: comparison of parametric analysis using MR- and CT-derived arterial input functions
}

\author{
Mihaela Rata $^{1}$ - David J. Collins ${ }^{1} \cdot$ James Darcy $^{1} \cdot$ Christina Messiou $^{1} \cdot$ Nina Tunariu ${ }^{1}$. \\ Nandita Desouza $^{1} \cdot$ Helen Young ${ }^{2} \cdot$ Martin O. Leach $^{1,3} \cdot$ Matthew R. Orton ${ }^{1}$
}

Received: 18 May 2015 / Revised: 7 August 2015 / Accepted: 3 September 2015 /Published online: 18 September 2015

(C) The Author(s) 2015. This article is published with open access at Springerlink.com

\begin{abstract}
Objectives Pharmacokinetic (PK) modelling of dynamic contrast-enhanced magnetic resonance imaging (DCE-MRI) data requires a reliable measure of the arterial input function (AIF) to robustly characterise tumour vascular properties. This study compared repeatability and treatment-response effects of DCE-MRI-derived PK parameters using a populationaveraged AIF and three patient-specific AIFs derived from pre-bolus MRI, DCE-MRI and dynamic contrast computed tomography (DC-CT) data.

Methods The four approaches were compared in 13 patients with abdominal metastases. Baseline repeatability [Bland-Altman statistics; coefficient of variation $(\mathrm{CoV})]$, cohort percentage change and $p$ value (paired $t$ test) and number of patients with significant DCE-MRI parameter change post-treatment (limits of agreement) were assessed.

Results Individual AIFs were obtained for all 13 patients with pre-bolus MRI and DC-CT-derived AIFs, but only 10/13 patients had AIFs measurable from DCE-MRI data. The best $\mathrm{CoV}$ $(7.5 \%)$ of the transfer coefficient between blood plasma and extravascular extracellular space $\left(K^{\text {trans }}\right)$ was obtained using a population-averaged AIF. All four AIF methods detected significant treatment changes: the most significant was the
\end{abstract}

Martin O. Leach

Martin.Leach@icr.ac.uk

CR-UK Cancer Imaging Centre, Division of Radiotherapy and Imaging, Institute of Cancer Research and Royal Marsden Hospital, London, UK

2 Early Clinical Development, AstraZeneca, Macclesfield, Cheshire, UK

3 CRUK Cancer Imaging Centre, MRI Unit, Royal Marsden Hospital, Downs Road, Sutton, Surrey SM2 5PT, UK
DC-CT-derived AIF. The population-based AIF was similar to or better than the pre-bolus and DCE-MRI-derived AIFs. Conclusions A population-based AIF is the recommended approach for measuring cohort and individual effects since it has the best repeatability and none of the PK parameters derived using measured AIFs demonstrated an improvement in treatment sensitivity.

Key Points

- Pharmacokinetic modelling of DCE-MRI data requires a reliable measure of AIF.

- Individual MRI-DCE-derived AIFs cannot reliably be extracted from patients.

- All four AIF methods detected significant $K^{\text {trans }}$ changes after treatment.

- A population-based AIF can be recommended for measuring cohort treatment responses in trials.

Keywords Magnetic resonance imaging · Computed tomography · Drug evaluation · Clinical trials, phase 1 . Comparative study

\begin{tabular}{ll}
\multicolumn{2}{l}{ Abbreviations } \\
DCE-MRI & $\begin{array}{l}\text { Dynamic contrast-enhanced magnetic } \\
\text { resonance imaging }\end{array}$ \\
PK & Pharmacokinetic \\
AIF & Arterial input function \\
PB & Pre-bolus \\
DC-CT & Dynamic contrast-computed tomography \\
POP & Population \\
$K^{\text {trans }}$ & Transfer coefficient between blood plasma \\
& and extravascular extracellular space \\
$v_{\mathrm{e}}$ & Volume of extravascular extracellular space \\
$v_{\mathrm{p}}$ & Volume of vascular plasma space \\
FFE & Fast-field echo
\end{tabular}




$\begin{array}{ll}\text { TR } & \text { Repetition time } \\ \text { TE } & \text { Echo time } \\ \text { FoV } & \text { Field of view } \\ \text { NSA } & \text { Number of sampling acquisitions } \\ \text { iPAT } & \text { Integrated parallel acquisition techniques } \\ \text { FA } & \text { Flip angle } \\ \text { CoV } & \text { Coefficient of variation } \\ \text { LoA } & \text { Limits of agreement } \\ \text { Hct } & \text { Haematocrit }\end{array}$

\section{Introduction}

Dynamic contrast-enhanced magnetic resonance imaging (DCE-MRI) is emerging as an imaging tool for diagnosis and assessing tumour response to novel anticancer antiangiogenic therapies [1-4]. The technique is based on the analysis of the temporal bio-distribution of an intravenously injected Gadolinium-based contrast agent within the imaged tumour. Pharmacokinetic (PK) modelling of such data requires a reliable measure of the arterial input function (AIF) in order to obtain robust estimates of physiological parameters characterising tumour vascular properties. The AIF represents the time course of the contrast concentration in the blood plasma pool, and its accuracy will strongly influence the output of the PK modelling. Therefore, a patient-specific measurement of the AIF per visit from a vessel feeding the tumour would in principle be a suitable approach. In practice, however, such direct measurements are heavily influenced by partial volume effects (especially for small vessels), in-flow effects, non-linear signal response, non-uniform $B_{1}$ field, pulsatile flow and other confounding factors. Variations due to these effects can be minimised by using a fixed AIF, which can reasonably be derived by averaging measured AIFs over a suitable population. It is plausible that where a cohort response is of interest (e.g. in a clinical trial), a population-averaged AIF may be appropriate, whereas when measuring individual responses, a measured AIF may be more suitable. This work aims to explore these two hypotheses. The repeatability and treatment-response effects of DCE-MRI-derived PK parameters are compared using AIFs derived in four ways:

1. Fixed AIF $\left(\mathrm{AIF}_{\mathrm{POP}}\right)[5]$

2. Individual AIFs derived from pre-bolus MRI data $\left(\mathrm{AIF}_{\mathrm{PB}}\right)$ $[6,7]$

3. Individual AIFs obtained from DCE-MRI data $\left(\mathrm{AIF}_{\mathrm{DCE}}\right.$ ) [8]

4. Individual AIFs derived from dynamic contrast computed tomography (DC-CT) data ( $\mathrm{AIF}_{\mathrm{CT}}$ ) (acquired same day as DCE-MRI)

The extended Kety model $[9,10]$ was used to model uptake of contrast in tumour tissues for all four AIF approaches. Baseline repeatability and treatment effect results were investigated in a subset of a cohort of patients previously shown to have a significant response to Cediranib in an early-phase trial [11].

\section{Materials and methods}

\section{Patient cohort}

Data presented here relate to a coherent and consistent subset of patients from an early-stage clinical trial comparing the efficacy of DCE-MRI and DC-CT for measuring treatment effects due to vascular-targeted therapies. This early-stage trial [11] investigated the treatment effects of Cediranib -a potent inhibitor of vascular endothelial growth factor receptor tyrosine kinases- and was shown to cause a cohort decrease in $K^{\text {trans }}$, the transfer coefficient between blood plasma and extravascular extracellular space. To ensure a homogeneous cohort, only coronal MR acquisitions of patients with at least three longitudinal scans were assessed. No particular criteria, other than the usual clinical inclusion/exclusion conditions defined within the trial protocol [11], were further considered here.

As a result, our cohort consisted of 13 patients with abdominal tumours (12 liver and one left para-aortic lymph node) imaged longitudinally at three time points. The cohort comprised seven women and six men, with ages ranging from 31 to 73 (mean 56.6) years. All patients were scanned twice, 7 days apart, prior to treatment, to assess repeatability of the methods. The third scan was performed after a minimum of 7 days of Cediranib therapy to investigate treatment changes. Patients gave written informed consent, and the local ethical committee approved the research study.

\section{MR acquisition protocol}

All 13 patients were imaged coronally on a $1.5 \mathrm{~T}$ Avanto Siemens scanner using a sequential breath-hold technique optimised for liver lesions [12]. The MR scanning consisted of a pre-bolus acquisition (one tenth of the standard dose; i.e. $0.02 \mathrm{ml} / \mathrm{kg}$ Magnevist contrast agent), followed by the main DCE acquisition at the standard dose of $0.2 \mathrm{ml} / \mathrm{kg}$ Magnevist. Both contrast doses were delivered by power injector at $3 \mathrm{ml} /$ $\mathrm{s}$, followed by $20 \mathrm{ml}$ of saline solution delivered at $2 \mathrm{ml} / \mathrm{s}$. The pre-bolus protocol was: 2D fast-field echo (FFE) sequence, a single slice $20 \mathrm{~mm}$ thick, repetition time/echo time $(\mathrm{TR} / \mathrm{TE})=$ $5.5 / 1.21 \mathrm{~ms}$, field of view $(\mathrm{FoV})=440 \times 440 \mathrm{~mm}^{2}, 128 \times 128$ matrix, temporal resolution $0.7 \mathrm{~s} /$ image, 60 pre-contrast images at $3^{\circ}$ and 340 dynamics at $20^{\circ}$. The corresponding parameters for the main DCE acquisition were 3D FFE sequence, 14 slices $5-\mathrm{mm}$ thick, TR/TE $=3.05 / 0.89 \mathrm{~ms}, \mathrm{FoV}=308 \times 380 \mathrm{~mm}^{2}$, $208 \times 256$ matrix, number of sampling acquisitions $(\mathrm{NSA})=1$, integrated parallel acquisition techniques $(\mathrm{PAT})=2$. Dynamic images with a flip angle (FA) of $16^{\circ}$ were acquired 
at $3 \mathrm{~s} /$ volume, obtained as pairs ( $6 \mathrm{~s}$ breath-hold at expiration), with 6-s breathing interval between each pair, and total acquisition time $4 \mathrm{~min}$. These were preceded by a calibration scan acquired in a single breath-hold (expiration) with the same parameters except $\mathrm{FA}=3^{\circ}$ and $\mathrm{NSA}=4$ to enable contrast quantification.

\section{CT acquisition protocol}

Corresponding DC-CT data were acquired on the same day no more than $4 \mathrm{~h}$ prior to MR scanning. Axial CT data were acquired with the following setup: GE Lightspeed; $0.5 \mathrm{ml} / \mathrm{kg}$ contrast agent (Omnipaque 300) followed by $20 \mathrm{ml}$ saline (both at $3-5 \mathrm{ml} / \mathrm{s}$ ); 5 -s delay followed by breath-hold cine covering $4 \times 5 \mathrm{~mm}^{2}$, at $0.5 \mathrm{~s} /$ volume in the centre of the lesion of interest over $55 \mathrm{~s}$ at $120 \mathrm{kV}$ and $60 \mathrm{~mA}$. Following this were 12 breath-hold acquisitions at 10 -s intervals.

\section{AIF data analysis}

\section{Pre-bolus MRI data $\left(A I F_{P B}\right)$}

Individual AIFs were extracted from the single-slice pre-bolus data for all patients at each of the three visits. $\mathrm{AIF}_{\mathrm{PB}}$ was measured from the abdominal aorta, a region of the aorta less affected by the pulsatile effects of the heart $[13,14]$. A fourparameter AIF model was used to fit the pre-bolus AIF data and a corresponding full-dose AIF was obtained by correcting for the duration and volume of the injection of the main DCE measurement [15]. The measured AIF (whole blood) was converted to contrast concentration in plasma assuming a haematocrit of 0.42 [5].

\section{DCE-MRI data $\left(A I F_{D C E}\right)$}

Individual AIFs from the main DCE-MRI data were obtained from a vessel visible in the acquired $\mathrm{FoV}$, which was the aorta in all cases. The same four-parameter AIF model was used to fit the visit-specific AIF $_{\mathrm{DCE}}$.

\section{$D C-C T$ data $\left(A I F_{C T}\right)$}

The DC-CT derived AIF was also obtained from the aorta, and a model-based correction method was used to account for the different contrast agents, delivery volumes and delivery rates for the DCE-MRI and DC-CT acquisitions (see "Appendix").

\section{Fixed AIF $\left(A I F_{P O P}\right)$}

The fixed AIF was based on the population-averaged AIF presented in [5].

\section{PK analysis of DCE-MRI tumour data}

Tumour regions of interest (ROI) from four central slices of the DCE-MRI data were fitted with the extended Kety model for each of the available four categories of AIF. Estimates of $K^{\text {trans }}$, volume of extravascular extracellular space $\left(v_{\mathrm{e}}\right)$ and volume of vascular plasma space $\left(v_{\mathrm{p}}\right)$ were obtained, and the median $\left(K^{\text {trans }}, v_{\mathrm{e}}\right)$ or mean $\left(v_{\mathrm{p}}\right)$ over the tumour was assessed for repeatability and treatment effects.

\section{Statistical analysis}

All statistics were calculated using log-transform of the raw values [16] and back-transformed appropriately to give percentage values. Baseline repeatability was calculated for $K^{\text {trans }}, v_{\mathrm{e}}$ and $v_{\mathrm{p}}$ estimates using the Bland-Altman statistic [17], expressed as coefficient of variation, $\mathrm{CoV}=100 \% \times\left[\exp \left(\sigma^{2} / 2\right)-1\right]^{1 / 2}$, where $\sigma^{2}$ is the variance of difference between baseline values. The limits of agreement (LoA) were derived from the BlandAltman variance $\sigma^{2}$ using $\operatorname{LoA}=100 \% \times \exp ( \pm 1.96 \sigma)$, which gives the range of changes in one patient that are within the $95 \%$ limits of the measurement repeatability. Patients with treatment changes outside the LoA were considered to have an individually significant effect, and the number of patients with changes outside the LoA was recorded for each measure. Treatment changes were computed as the difference between posttreatment values and the mean of the two pre-treatment values.

Table 1 Repeatability and treatment effects for three main pharmacokinetic (PK) parameters as measured from our comparative analysis of arterial input function (AIF) methods

\begin{tabular}{|c|c|c|c|c|c|c|}
\hline & \multirow[t]{2}{*}{ AIF } & \multirow{2}{*}{$\begin{array}{l}\text { Number of } \\
\text { patients }\end{array}$} & \multirow{2}{*}{$\begin{array}{l}\mathrm{CoV} \\
(\%)\end{array}$} & \multicolumn{3}{|c|}{ Treatment effects } \\
\hline & & & & (\% decrease) & $P$ value & $\begin{array}{l}\text { No. outside } \\
\text { LoA }\end{array}$ \\
\hline \multirow[t]{4}{*}{ Ktrans } & POP & 13 & 7.5 & 49.5 & 0.023 & 11 \\
\hline & DCE & 10 & 23.7 & 57.0 & 0.043 & 6 \\
\hline & PB & 13 & 21.0 & 65.7 & 0.021 & 10 \\
\hline & $\mathrm{CT}$ & 13 & 11.8 & 58.9 & 0.0049 & 11 \\
\hline \multirow[t]{4}{*}{$\mathrm{v}_{\mathrm{e}}$} & POP & 13 & 11.0 & 32.6 & 0.082 & 4 \\
\hline & DCE & 10 & 10.9 & 47.9 & 0.048 & 6 \\
\hline & PB & 13 & 22.4 & 45.3 & 0.18 & 5 \\
\hline & $\mathrm{CT}$ & 13 & 18.4 & 36.7 & 0.041 & 5 \\
\hline \multirow[t]{4}{*}{$v_{p}$} & POP & 13 & 62.5 & 29.7 & 0.064 & 0 \\
\hline & DCE & 10 & 50.4 & 14.5 & 0.6 & 0 \\
\hline & PB & 13 & 86.8 & 48.0 & 0.021 & 0 \\
\hline & $\mathrm{CT}$ & 13 & 54.2 & 37.4 & 0.085 & 1 \\
\hline
\end{tabular}

$P O P$ population, $D C E$ dynamic contrast enhanced, $P B$ pre-bolus, $C T$ computed tomography, $L O A$ limits of agreement, $C o V$ coefficient of variation, $K^{\text {trans }}$ transfer coefficient between blood plasma and extravascular extracellular space, $v_{\mathrm{e}}$ volume of extravascular extracellular space, $v_{p}$ volume of vascular plasma space 
The mean cohort changes were computed and back-transformed to give percentage changes; $p$ values were obtained using two-sided paired $t$ tests on the log-transformed values for $K^{\text {trans }}$, $v_{\mathrm{e}}$ and $v_{\mathrm{p}}$, and also for AIF parameters (see "Appendix") for all three measured AIFs.

\section{Results}

The main results are presented in Table 1 and Fig. 1 for the three PK parameters.

Measured AIFs were extracted for all 13 patients from both the pre-bolus MR data and the DC-CT data. No measurable vessel was present in the DCE-MRI data in 3/13 patients, so no tissue parameters were obtained for this AIF approach in these patients.

The best $K^{\text {trans }}$ repeatability was $7.5 \%$, which was achieved when using a population-derived $\mathrm{AIF}_{\mathrm{POP}}$, whereas those obtained with measured $\mathrm{AIF}_{\mathrm{PB}}$ and $\mathrm{AIF}_{\mathrm{DCE}}$ were both $>20 \%$. At $11.8 \%, \mathrm{AIF}_{\mathrm{CT}}$ had a smaller $\mathrm{CoV}$ for $K^{\text {trans }}$ than using either of the MR-measured AIFs.
Post-treatment $K^{\text {trans }}$ cohort decreases ranged from $49.5 \%$ $\left(\mathrm{AIF}_{\mathrm{POP}}\right)$ to $65.7 \%\left(\mathrm{AIF}_{\mathrm{PB}}\right)$ and all were statistically significant $(p<0.05)$, with the most significant being the $\operatorname{AIF}_{\mathrm{CT}}(p=$ 0.0049). All AIF methods identified significant individual responders: $\mathrm{AIF}_{\mathrm{POP}}$ and $\mathrm{AIF}_{\mathrm{CT}}$ identified $11 / 13$ patients with $K^{\text {trans }}$ decreases outside the LoA; $\mathrm{AIF}_{\mathrm{PB}}$ identified 10/13; $\mathrm{AIF}_{\mathrm{DCE}}$ only identified 6/13.

Similar repeatability ranges were obtained for $v_{\mathrm{e}}(10.9-$ $22.4 \%$ ), while significantly worse repeatability was obtained for $v_{\mathrm{p}}(50.4-86.8 \%)$. For both $v_{\mathrm{e}}$ and $v_{\mathrm{p}}$, the best CoV was with $\mathrm{AIF}_{\mathrm{DCE}}$ and the worst was with $\mathrm{AIF}_{\mathrm{PB}}$. Posttreatment $v_{\mathrm{e}}$ cohort decreases were in the range 32.6$47.9 \%$, but only $\mathrm{AIF}_{\mathrm{DCE}}$ and $\mathrm{AIF}_{\mathrm{CT}}$ reached significance $(p<0.05)$, while approximately half of the patients (4-6/13) had individual reductions outside the LoA. Only $\mathrm{AIF}_{\mathrm{PB}}$ identified a significant cohort decrease in $v_{\mathrm{p}}$ $(p=0.021)$.

Typical patient AIF curves as derived from the four methods are shown in Fig. 2. The only significant treatment changes to AIF parameters were for $\mathrm{AIF}_{\mathrm{CT}}$, where the amplitude of the recirculation phase $a_{\mathrm{R}}$ increased by $66 \%(p=$ $0.001)$, and its duration $\mu_{\mathrm{R}}$ decreased by $13 \%(p=0.04)$.
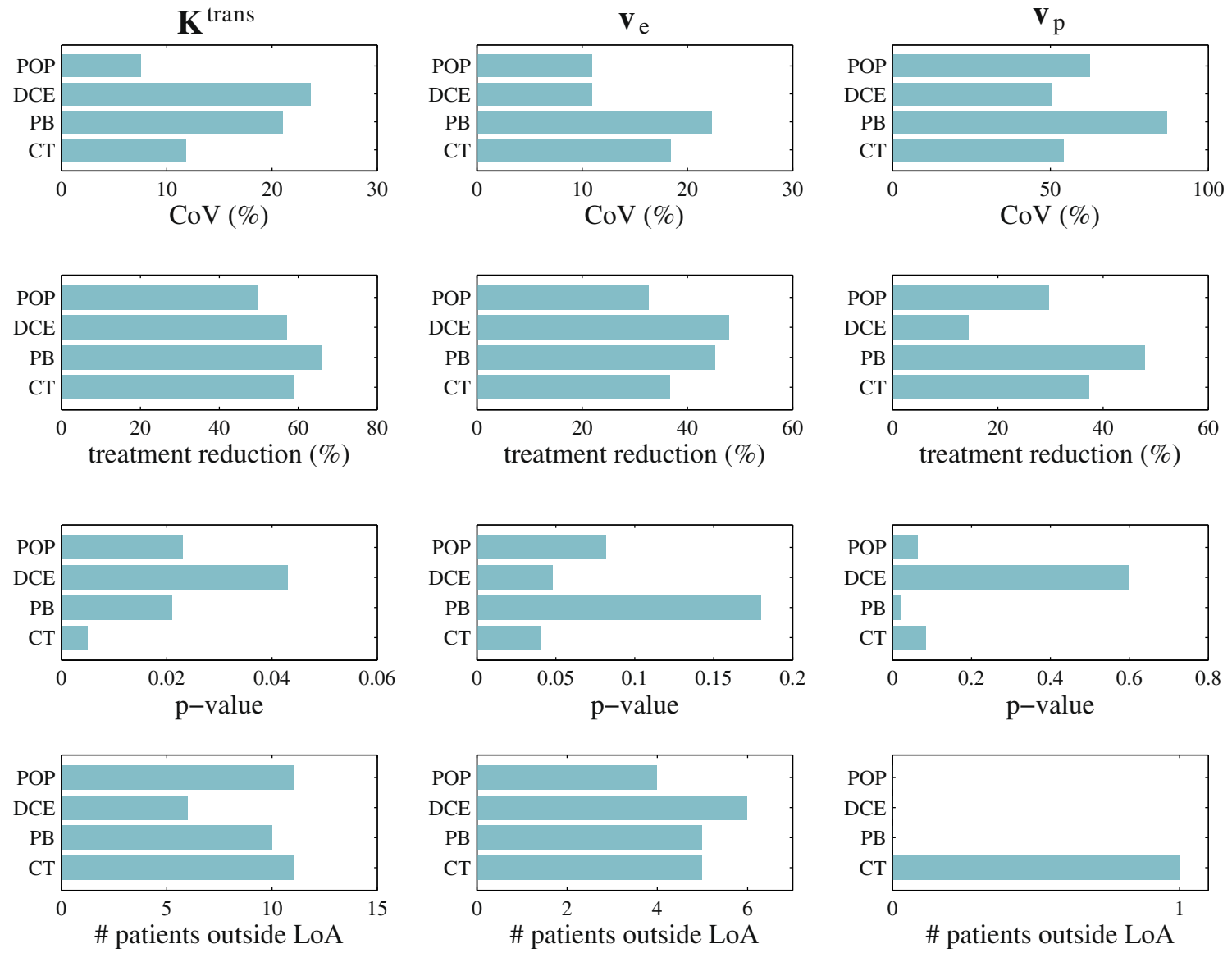

Fig. 1 Representation of main results presented in Table 1. POP population, $D C E$ dynamic contrast enhanced, $P B$ pre-bolus, $C T$ computed tomography, LoA limits of agreement, $\mathrm{CoV}$ coefficient of

variation, $K^{\text {trans }}$ transfer coefficient between blood plasma and extravascular extracellular space, $v_{\mathrm{e}}$ volume of extravascular extracellular space, $v_{p}$ volume of vascular plasma space 

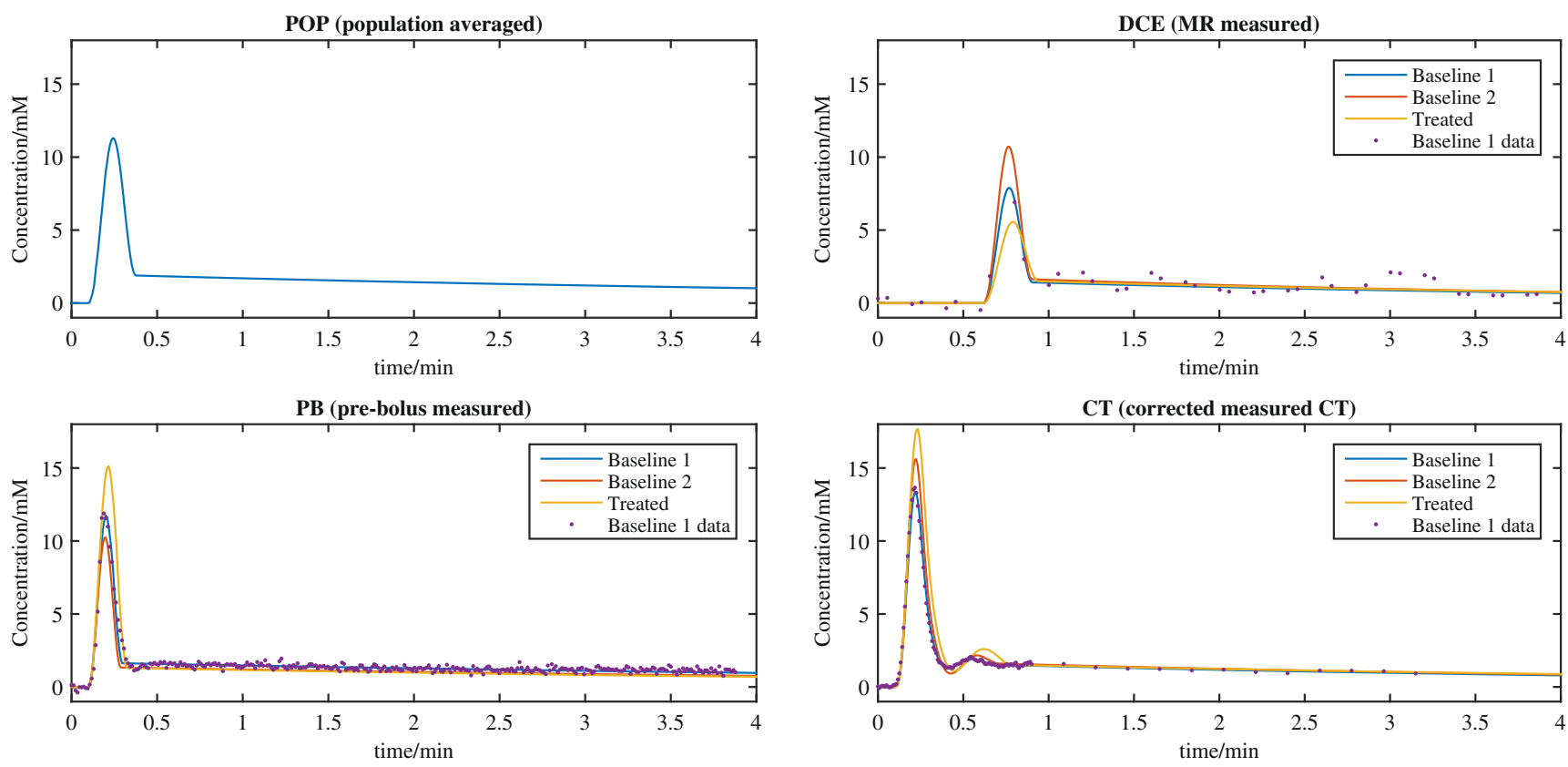

Fig. 2 Typical arterial input function (AIF) curves for one patient as derived from the four methods compared. Fits of baselines and post-treatment curves together with the measured AIF curve for baseline

Treatment changes for all parameters of $\mathrm{AIF}_{\mathrm{DCE}}$ and $\mathrm{AIF}_{\mathrm{PB}}$ and the remaining parameters for $\mathrm{AIF}_{\mathrm{CT}}$ had $p>0.1$.

\section{Discussion}

Whilst using a DC-CT AIF is not a feasible approach in general, the good $K^{\text {trans }}$ repeatability using $\mathrm{AIF}_{\mathrm{CT}}$ is remarkable, since DC-CT data were acquired up to $4 \mathrm{~h}$ before DCE-MRI data and involved a different contrast agent and imaging approach. Nevertheless, the net effect of these additional sources of variability still results in a lower $K^{\text {trans }}$ variability with $\mathrm{AIF}_{\mathrm{CT}}$ than with $\mathrm{AIF}_{\mathrm{DCE}}$ or $\mathrm{AIF}_{\mathrm{PB}}$, both of which were obtained during the same imaging session as the main DCE-MRI measurement. This suggests that, in principle, using a measured AIF can provide higher accuracy of parametric values but that significant improvements to AIF measurement using DCE-MRI are needed to yield an overall reduction in variability by accounting for genuine physiological AIF variability.

All four AIF methods detected similar $K^{\text {trans }}$ decreases on treatment, and whilst all four were significant $(p<0.05), \mathrm{AIF}_{\mathrm{CT}}$ was an order of magnitude more significant than the other three $(p=0.0049)$. In contrast, $\mathrm{AIF}_{\mathrm{DCE}}$ identified only $6 / 13$ patients with $K^{\text {trans }}$ decreases outside the LoA, compared with $10 / 13$ or $11 / 13$ patients identified when using the other three AIFs. Taken together, the $K^{\text {trans }}$ repeatability and treatment changes observed with $\mathrm{AIF}_{\mathrm{CT}}$ indicate that, in principle, visit-specific AIFs would be preferred, but the results with $\mathrm{AIF}_{\mathrm{DCE}}$ and $\mathrm{AIF}_{\mathrm{PB}}$ suggest that, in practice, neither of these approaches a significantly better performance than a population-averaged AIF. In addition, 3/13

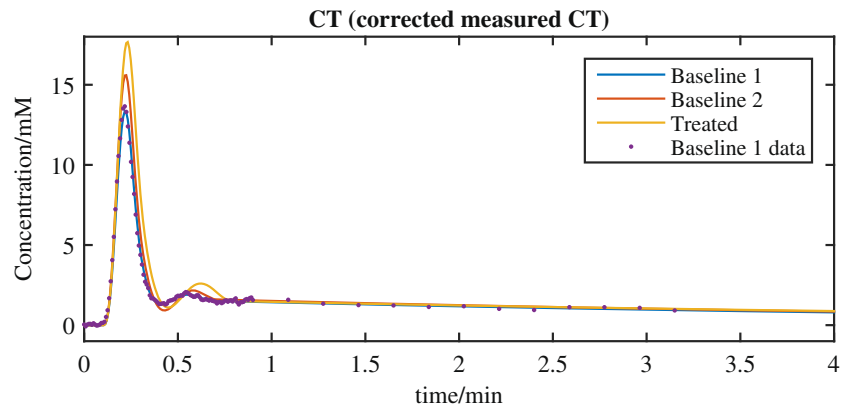

1 data are presented for each measurement technique. $P O P$ population, $D C E$ dynamic contrast enhanced, $M R$ magnetic resonance, $P B$ pre-bolus, CT computed tomography

cases did not yield a useable AIF from DCE-MRI data due to tumour location, which is a significant practical limitation.

Further comparisons between the various AIFs can be made from the $v_{\mathrm{e}}$ and $v_{\mathrm{p}}$ statistics in Table 1 and Fig. 1. The repeatability of $v_{\mathrm{e}}$ shows no difference between $\mathrm{AIF}_{\mathrm{POP}}$ and $\mathrm{AIF}_{\mathrm{DCE}}$, whilst that for $\mathrm{AIF}_{\mathrm{PB}}$ is around twice as large. $\mathrm{AIF}_{\mathrm{DCE}}$ gives a $v_{\mathrm{e}}$ treatment $p$ value of borderline significance, whilst $\mathrm{AIF}_{\mathrm{POP}}$ and $\mathrm{AIF}_{\mathrm{PB}}$ are not significant, indicating $\mathrm{AIF}_{\mathrm{DCE}}$ may be the best approach if only $v_{\mathrm{e}}$ is of interest. None of the AIF approaches yields a $v_{\mathrm{p}}$ repeatability suitable for monitoring individual or cohort changes.

There were no significant treatment changes to AIF parameters for $\mathrm{AIF}_{\mathrm{DCE}}$ or $\mathrm{AIF}_{\mathrm{PB}}$, whilst the significant changes to $\mathrm{CT}$-measured AIFs are associated with the amplitude and duration of the recirculation phase $\left(a_{\mathrm{R}}\right.$ and $\left.\mu_{\mathrm{R}}\right)$, which is not visible in MR-measured AIFs. Although these changes may be genuine, it is more likely that the significant $p$ values obtained are due to statistical fluctuations, especially since the $\mathrm{AIF}_{\mathrm{CT}}$ model contains eight parameters.

A population haematocrit (Hct) of 0.42 was assumed in this study. Preliminary tests (data not shown here) investigated Hct variability from two baseline measurements over a cohort of 21 patients. The repeatability coefficient was $3.4 \%$, suggesting a minimal impact of Hct variability on the $K^{\text {trans }}$ estimate (since $K^{\text {trans }}$ is approximately proportional to $1-\mathrm{Hct}$ ).

When assessing treatment response, an AIF measured per session would account for any changes in AIF and, in principle, this should lead to lower variability and, by extension, to improved sensitivity and specificity of $K^{\text {trans }}$ changes to a tumour effect. This specificity is particularly important if 
treatment has an effect on the AIF, since in such cases a population-derived AIF would mean $K^{\text {trans }}$ treatment changes would be confounded by AIF changes. In this study, no significant MRI AIF changes were detected, which is consistent with the finding that the population-derived AIF had the best overall performance in these data. Since AIF variability and changes with treatment have not previously been reported, it is unclear whether this is typical where DCE-MRI measurements are used. Therefore, given the poor performance of both MRbased AIF measurements, we suggest that using a populationderived AIF is the most appropriate method when measuring cohort treatment responses for DCE-MRI clinical trials unless there is good evidence that the treatment could affect the AIF.

Our findings reinforce the common practice of using a population-based AIF for quantitative DCE-MRI clinical trials when assessing tumour response [2,5]. Other clinical studies have compared DCE-MRI metrics when using a population AIF (or cohort-averaged AIF) versus different approaches of measuring individual AIFs for various tumour sites, e.g. abdomen [5], osteosarcomas [18], neck [19, 20], prostate [21] or breast [22]. Overall, these studies reported no significant difference/improvement in PK estimates when measuring individual AIFs compared with a population AIF, which is in agreement with our conclusion.

Acknowledgments We are grateful for patients' availability and cooperation during the course of this study.

The scientific guarantor of this publication is Prof Martin Leach. The authors of this manuscript declare no relationships with any companies, whose products or services may be related to the subject matter of the article. This work was supported by funding from CRUK and EPSRC support to the Cancer Imaging Centre at ICR and RMH in association with MRC and the Department of Health C1060/A10334, C1060/ A16464 and NHS funding to the NIHR Biomedical Research Centre and the Clinical Research Facility in Imaging. An Experimental Cancer Medicine Centre Network award (joint initiative, CRUK and UK Department of Health) [grants C51/A7401 \& C12540/A15573]). This work was also supported by AstraZeneca. Support was also received from EPSRC Platform Grant EP/H046526/1. MOL is an NIHR senior investigator. No complex statistical methods were necessary for this paper. Institutional Review Board approval was obtained. Written informed consent was obtained from all patients. Some study patients or cohorts were previously reported in Messiou C, Orton M, Ang JE, et al. (2012) Advanced solid tumors treated with Cediranib: comparison of dynamic contrast-enhanced MR imaging and CT as markers of vascular activity. Radiology 265(2):426-436.

Methodology: retrospective, experimental, performed at one institution.

\section{Appendix}

AIF correction methods used to give an AIF that is appropriate for fitting DCE-MRI tissue data to account for volume and delivery rates used with pre-bolus and DC-CT-measured AIFs, and also for the different contrast agents used for DC-CT measurement. In all cases, the contrast agent is delivered using a power injector at a known constant rate so that the temporal profile of the injection is a rectangular pulse, $R_{\mathrm{IN}}(t)$, with duration equal to the injected volume divided by the delivery rate and amplitude set to unity (scaling will be considered in more detail below). Signals measured with DC-CT or with the prebolus can therefore be expressed as the convolution of $R_{\mathrm{IN}}(t)$ with a vascular impulse response, $V(t)$, that is $S(t)=V(t) \otimes R_{\mathrm{IN}}\left(t-t_{0}\right)$,

where $S(t)$ is the modelled signal and $t_{0}$ is the injection delay relative to the acquisition timing. The vascular impulse response is assumed to be independent of the contrast agent and injection volume and rate, so the AIF used to fit the DCE-MRI tissue data can be inferred using

$A I F(t)=V(t) \otimes R_{\mathrm{DCE}}(t)$,

where $R_{\mathrm{DCE}}(t)$ is a rectangular pulse with duration corresponding to the DCE-MRI injection rate and volume.

The amplitude of $R_{\mathrm{DCE}}(t)$ accounts for the different volumes and contrast properties of the agents used in each measurement and for any signal scaling. For the pre-bolus measurement, $S(t)$ is the whole-blood contrast concentration after $\mathrm{T}_{1}$ quantification of the double flip-angle data, and since the contrast agent and injection rate are the same for the pre-bolus and the main DCE-MRI acquisition, the amplitude scaling is $1 /(1-\mathrm{Hct})$ so that the AIF is for the plasma concentration. For DC-CT measurement, $S(t)$ is the measured signal in Hounsfield units (HU), and the scaling on $R_{\mathrm{DCE}}(t)$ is given by $0.0507 \mathrm{mmol} / \mathrm{l} / \mathrm{HU}$. This is derived with the formula $S_{\mathrm{CT}} \times$ $D_{\mathrm{MR}} \div D_{\mathrm{CT}} \div(1-\mathrm{Hct})$, where:

1. $S_{\mathrm{CT}}=0.347 \mathrm{mmol} / \mathrm{l} / \mathrm{HU}$ is the molarity scaling factor between iodine concentration and HU measured with $\mathrm{CT}$ (obtained from phantom measurements)

2. $D_{\mathrm{MR}}=0.1 \mathrm{mmol} / \mathrm{kg}$ is the molar dose of MR contrast agent (corresponding to $0.2 \mathrm{ml} / \mathrm{kg}$ of Magnevist at $0.5 \mathrm{mmol} / \mathrm{ml})$

3. $D_{\mathrm{CT}}=1.18 \mathrm{mmol} / \mathrm{kg}$ is the molar dose of CT contrast agent (corresponding to $0.5 \mathrm{ml} / \mathrm{kg}$ of Omnipaque 300 at $300 \div$ $126.9 \mathrm{mmol} / \mathrm{ml}$; Omnipaque 300 has $300 \mathrm{mg}$ iodine per millilitre and iodine has atomic weight $126.9 \mathrm{mg} / \mathrm{mmol}$ )

4. $\mathrm{Hct}=0.42$ is the hematocrit [5]

The DC-CT vascular impulse response is modelled using an eight-parameter model [23]

$$
\begin{aligned}
V(t)= & a_{\mathrm{B}} V_{\mathrm{C}}\left(t, \mu_{\mathrm{B}}\right) \otimes\left(\mu_{\mathrm{M}} e^{-\mu_{\mathrm{M}} t}\right)+a_{\mathrm{R}} V_{\mathrm{C}}\left(t-\tau_{\mathrm{R}}, \mu_{\mathrm{R}}\right) \\
& +a_{\mathrm{E}} V_{\mathrm{C}}\left(t-\tau_{\mathrm{R}}, \mu_{\mathrm{R}}\right) \otimes\left(e^{-\mu_{\mathrm{E}} t}\right),
\end{aligned}
$$

where $V_{\mathrm{C}}(t, \mu)=1-\cos (\mu t)$ for $0<t<2 \pi / \mu$ and zero elsewhere [15]. The first term describes the first-pass phase of the 


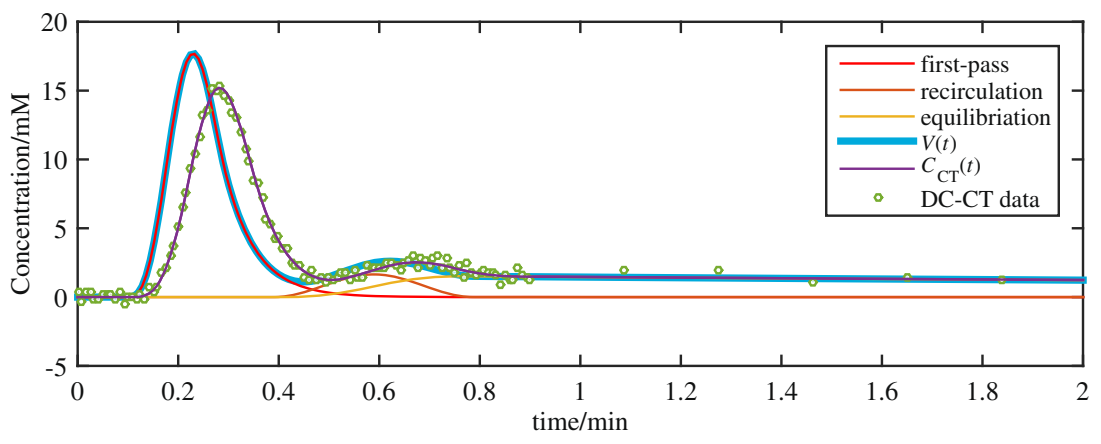

Fig. 3 Vascular impulse response model showing first-pass, recirculation and washout components and total vascular impulse response $V(t)$. These impulse response curves correspond to the data shown and are obtained by least-squares fitting of $C_{\mathrm{T}}(t)$ (which includes the effect of the known rectangular injection curve) to the measured dynamic contrast computed tomography (DC-CT) data response with $V_{\mathrm{C}}\left(t, \mu_{\mathrm{B}}\right)$ modelling the dispersion of contrast passing through the heart and $\mu_{\mathrm{M}} e^{-\mu_{\mathrm{M}} t}$ the passage through the lungs. The second term describes the recirculation phase of the response. The third term describes the equilibration phase of the response. The utility of this model is that it accurately captures all features observed in the DC-CT data, and all the convolutions required to compute $\mathrm{AIFs}$ and the tissue response curves (using the extended Kety model) can be evaluated analytically, leading to rapid and robust data fitting.

For the pre-bolus data, the recirculation phase is not visible (Fig. 2), and so a simplified form of the vascular impulse response with four parameters only is used:

$V(t)=a_{\mathrm{B}} V_{\mathrm{C}}\left(t, \mu_{\mathrm{B}}\right)+a_{\mathrm{E}} V_{\mathrm{C}}\left(t, \mu_{\mathrm{B}}\right) \otimes\left(e^{-\mu_{\mathrm{E}} t}\right)$

where the first term describes the first-pass phase and the second term the equilibration phase. This model is also used to fit the AIF obtained from DCE-MRI data, but as this is the same acquisition as the tumour data, there is no need to correct for injection duration; the only adjustment needed is to divide by (1-Hct) to give a plasma concentration.

The overall scheme for using the DC-CT/pre-bolus AIF measurements with the DCE-MRI tissue data is:

1. Fit the DC-CT/prebolus data with $S(t)$ using a leastsquares estimation routine. The estimated parameters are $a_{\mathrm{B}}, \mu_{\mathrm{B}}, \mu_{\mathrm{M}}, a_{\mathrm{R}}, \mu_{\mathrm{R}}, \mathrm{t}_{\mathrm{R}}, a_{\mathrm{E}}, \mu_{\mathrm{E}}$ and $t_{0}$, for DC-CT data, and $a_{\mathrm{B}}, \mu_{\mathrm{B}}, a_{\mathrm{E}}, \mu_{\mathrm{E}}$ and $t_{0}$ for pre-bolus data, and the known $\mathrm{DC}-\mathrm{CT} /$ pre-bolus injection duration is used in the model equation for $S(t)$.

2. Fit the tissue data using the extended Kety model with the input function $A I F(t)$, which uses vascular impulse response parameters obtained in Step 1 with the DCEMRI injection duration information and scaling factor $0.0507 \mathrm{mmol} / \mathrm{l} / \mathrm{HU}$ for the DC-CT and $1 /(1-\mathrm{Hct})$ for the pre-bolus.
Open Access This article is distributed under the terms of the Creative Commons Attribution-NonCommercial 4.0 International License (http://creativecommons.org/licenses/by-nc/4.0/), which permits any noncommercial use, distribution, and reproduction in any medium, provided you give appropriate credit to the original author(s) and the source, provide a link to the Creative Commons license, and indicate if changes were made.

\section{References}

1. Padhani AR, Husband JE (2001) Dynamic contrast-enhanced MRI studies in oncology with an emphasis on quantification, validation and human studies. Clin Radiol 56:607-620

2. Leach MO, Brindle KM, Evelhoch JL et al (2005) The assessment of antiangiogenic and antivascular therapies in early-stage clinical trials using magnetic resonance imaging: issues and recommendations. Br J Cancer 92:1599-1610

3. O'Connor JP, Jackson A, Parker GJ, Jayson GC (2007) DCE-MRI biomarkers in the clinical evaluation of antiangiogenic and vascular disrupting agents. Br J Cancer 96:189-195

4. Leach MO, Morgan B, Tofts PS et al (2012) Imaging vascular function for early stage clinical trials using dynamic contrastenhanced magnetic resonance imaging. Eur Radiol 22:1451-1464

5. Parker GJ, Roberts C, Macdonald A et al (2006) Experimentallyderived functional form for a population-averaged high-temporalresolution arterial input function for dynamic contrast-enhanced MRI. Magn Reson Med 56:993-1000

6. Köstler H, Ritter C, Lipp M, Beer M, Hahn D, Sandstede J (2004) Prebolus quantitative MR heart perfusion imaging. Magn Reson Med 52:296-299

7. Kershaw LE, Cheng HL (2011) A general dual-bolus approach for quantitative DCE-MRI. Magn Reson Imaging 29:160-166

8. Jackson A, Haroon H, Zhu XP, Li KL, Thacker NA, Jayson G (2002) Breath-hold perfusion and permeability mapping of hepatic malignancies using magnetic resonance imaging and a first-pass leakage profile model. NMR Biomed 15:164-173

9. Kety SS (1951) The theory and applications of the exchange of inert gas at the lungs and tissues. Pharmacol Rev 3:1-41

10. Tofts PS (1997) Modeling tracer kinetics in dynamic Gd-DTPA MR imaging. J Magn Reson Imaging 7:91-101

11. Messiou C, Orton M, Ang JE et al (2012) Advanced solid tumors treated with cediranib: comparison of dynamic contrast-enhanced MR imaging and CT as markers of vascular activity. Radiology 265 : 426-436 
12. Orton MR, Miyazaki K, Koh DM et al (2009) Optimizing functional parameter accuracy for breath-hold DCE-MRI of liver tumours. Phys Med Biol 54:2197-2215

13. Rata M, Collins D, Darcy J et al (2012) Evaluation of reproducibility of measured arterial input functions and DCE-MRI-derived model estimates obtained using either prebolus individual AIF's or population derived AIF's. Proc Intl Soc Magn Reson Med 20: 237

14. Jajamovich GH, Calcagno C, Dyvorne HA, Rusinek H, Taouli B (2014) DCE-MRI of the liver: reconstruction of the arterial input function using a low dose prebolus contrast injection. PLoS One 9, e115667

15. Orton MR, d'Arcy JA, Walker-Samuel S et al (2008) Computationally efficient vascular input function models for quantitative kinetic modelling using DCE-MRI. Phys Med Biol 53:1225-1239

16. Keen ON (1995) The log transformation is special. Stat Med 14: 811-819

17. Bland JM, Altman DG (1986) Statistical methods for assessing agreement between two methods of clinical measurement. Lancet 1:307-310

18. Wang Y, Huang W, Panicek DM, Schwartz LH, Koutcer JA (2008) Feasibility of using limited-population-based arterial input function for pharma-cokinetic modeling of osteosarcoma dynamic contrast-enhanced MRI data. Magn Reson Med 59:11831189

19. Shukla-Dave A, Lee N, Stambuk H et al (2009) Average arterial input function for quantitative dynamic contrast enhanced magnetic resonance imaging of neck nodal metastases. BMC Med Phys 9:4

20. Onxley JD, Yoo DS, Muradyan N, MacFall JR, Brizel DM, Craciunescu OI (2014) Comprehensive population-averaged arterial input function for dynamic contrast-enhanced magnetic resonance imaging of head and neck cancer. Int J Radiat Oncol Biol Phys 89:658-665

21. Meng R, Chang SD, Jones EC, Goldenberg SL, Kozlowski $P$ (2010) Comparison between population average and experimentally measured Arterial Input Function in predicting biopsy results in prostate cancer. Acad Radiol 17:520-525

22. Li X, Welch EB, Arlinghaus LR et al (2011) A novel AIF tracking method and comparison of DCE-MRI parameters using individual and population-based AIFs in human breast cancer. Phys Med Biol 56:5753-5769

23. Miyazaki K, Jerome NP, Collins DJ et al (2015) Demonstration of the reproducibility of free-breathing diffusion-weighted MRI and dynamic contrast enhanced MRI in children with solid tumours: a pilot study. Eur Radiol. doi:10.1007/s00330-015-3666-7 